\title{
ICTs as a Distinct Detection Approach for Dyslexia Screening: A Contemporary View
}

\author{
https://doi.org/10.3991/ijoe.v15i13.11011
}

\author{
Athanasios S. Drigas ${ }^{(\varpi)}$, Stratigoula Politi-Georgousi \\ N.C.S.R. 'Demokritos', Athens, Greece \\ dreiit.demokritos.gr
}

\begin{abstract}
In contemporary times, various studies have been conducted around the world, researching the use of Information and Communication Technologies (ICTs) on dyslexia screening in all ages. Screening procedure constitutes the first vital step to investigate the kind of these experiencing difficulties, which sets the foundations for a well-designed intervention. Thus, ICTs is becoming an emerging unique supplementary strategy, using cost-effective and easy to access advanced tests for gaining in-depth knowledge about students' learning profiles. The purpose of this literature review is to present an indicative number of digital assessment tools (e.g. computerized systems, software, applications etc.) for detecting dyslexia, that have been designed or have been implemented on dyslexic population during the last thirteen years (20052018). The classification of proposed papers has been carried out according to the life stage, each of whom analyzed.
\end{abstract}

Keywords-Screening, ICTs, Dyslexia, assessment tools

\section{$1 \quad$ Introduction}

Dyslexia is an euro biologically based specific learning difficulty, which is distinguished by a peculiar balance of skills [1], [2]. Despite IQ, sufficient instruction and intact sensory abilities, dyslexic students exhibit problems related to spelling, fluent and/or accurate word recognition, as well as poor decoding abilities, which end up to a significant deficit in phonological aspect of language. [3], [1].

A matter, which frequently generates a great deal of heated debate is dyslexia screening due to the multidimensional nature of this disorder. Snowling (2013) broached the issue of understanding of dyslexia before a sense of failure set in, reporting that teacher assessments and measurements might be a credible screening solution [4]. Teachers are entirely responsible for instructing all dyslexic or non-dyslexic students without exception, affecting on methods, interactions and decisions related to dyslexia [5]. In this attempt, the use of a suitable assessment tool among innumerable tools and practices is considered a prerequisite for successful completion of this process.

These days, ICTs seem to be a major vehicle for both dyslexia screening and required interventions, which are adjusted in accordance with students' specific learning 
problems and needs. Indeed, ICTs are such an outstanding factor for improving the conventional dyslexia detection methods, as well as for triggering the exploration of new perspectives as far as the identification of dyslexic individuals is concerned [6]. Rooms (2000) referred to potential benefits by the ICT use for dyslexic students, highlighting that they:

- Are easy to access and available without making dyslexic students feel different or excluded

- Include multisensory approaches (aural, oral, visual, kinesthetic)

- Incorporate systems for improving these students' difficulties [7]

In addition, noteworthy findings of preceding studies reported that computerizedassessment process provides not only psychologists but also teachers with the opportunity to conduct screening of cognitive abilities and other vital skills in an easy and fast way. Apart from that, students predominantly are fervent supporters of this kind of screening [8].

For this purpose, numerous computational strategies assist and boost the effectiveness of the commonplace dyslexia screening procedures such as interactive multimedia, virtual environments, neural networks, software, fuzzy logic, game-based techniques and mobile applications [6], [9], [10], [11]. Each of them features sophisticated functions, which make assessment procedures more convenient.

Thus, the following question will be raised: Are ICTs an alternative profitable approach for facilitating dyslexia screening process. In the present article, a substantial number of different studies, referred to computerized screening dyslexia tools, were collected and were analyzed with the aim of inspecting the usability and benefits of this detection method. Classification of each test was directly dependent on the age the individual is evaluated for the difficulty that he/she experience.

\section{Dyslexia Screening at Preschool Stage}

Early identification of dyslexia constitutes an integral part of a fruitful intervention as research data indicated that children who were at risk for dyslexia made remarkable progress, when detected early and joined in an intervention program [12]. Thus, a great number of ICTs assessment tools have been developed to facilitate dyslexia screening process.

Specifically, Sood et al. (2017) invented a game-based digital application, that aimed at enhancing the early detection, monitoring and management of dyslexia ("DIMMAND") in young children. The digital tool is composed of a great diversity of groundbreaking games, which systematically explore a kid's abilities in disparate aspects of writing, reading, visual and auditory perception. This digital solution was developed in phases, as researchers, giving emphasis on the full functional specification of the games, constructed overall architecture, implemented some of them and interacted with adult dyslexics and immediate surroundings. Indeed, the initial step provided insights into the nature of disabilities faced by dyslexics and the teachers' 
management, triggering stakeholders to test successfully and confirmed the viability of this emerging cost-effective and efficient application [13].

In Malaysia, Mat et al. (2014) designed a Computer-based Dyslexia Screening Test (CDST). This application comprises five assessment modules and related design guidelines for identification of dyslexia at an early stage, which have to do with Reading, Mathematics, Spelling, Hearing and IQ. Every module, being easy and amusing, displays results independently at the end of activity. As highlighted, Computer-based Dyslexia Screening Test was considered to be a beneficial alternative way, that urged students with possible difficulties to take part in the activities, without them knowing they were being detected for dyslexia. However, further research was needed for the development of this new system through teachers' and parents' involvement [14].

Gaggi et al. (2012), also, constructed a system included by diverse serious games, that oriented to predict the potential signs of developing dyslexia in preschool children. Each of the games, training peculiar skills, utilizes both visual cue stimuli and distractors, which affect players' scores. This serious of games was tested, in order for its usability, robustness and correctness be confirmed, during all the stages of its development. Primarily, this experiment was carried out in a small group of children and a second group of kids was involved afterwards in Italy, playing the suggested games through necessary given instructions. Most of the children were advocate of the new system, declaring that they were entertaining and easy. Undisputedly, the promising results of the preliminary study proved that serious games provided a unique way to evaluate a high risk of dyslexia, taking into consideration the users' performance [15].

In India, Thulasi-Bai et al. (2016) proposed an online fine motor movements screening tool for dyslexia, called "RU-Lexic", so that they could assess kids with dyslexia at an early stage without much of manual intervention. "RU-Lexic" constitutes an automated multimedia instrument, that includes a great variety of screening tests to measure the fine motor movements in dyslexic toddlers through kinesthetic perception. In this project, the content and especially the activities of the web tool, related to evaluations, were structured, as a Dyslexic child was encouraged to complete the tasks, in order for its kinesthetic perception be recorded and analyzed. Undoubtedly, this web application was expected to be a user friendly, magnificent and reliable strategy for dyslexia screening, which aroused children's curiosity [16].

In the USA, Facoetti et al. (2014) suggested a set of multiplatform games for the detection of dyslexia in preschooler children, which was constructed at a preliminary research stage. These games are played in sequence, used both online and offline. The system includes a great number of games, which are intent to evaluate different possible symptoms of dyslexia. There is no doubt that serious games were expected to be a challenging and amusing system, which captured not only preschoolers' but also adults' attention. Nevertheless, supplementary tests were needed to be conducted in preschoolers, so as for its reliability to be validated [17].

In Belgium, Den-Audenaeren et al. (2013) designed a game-based application, known as "DYSL-X", aiming to identify possible signs of dyslexia at preschool stage. This tablet game includes different tests in the form of unique games with incorporated automated measurements and scoring to assess players' performance. Five-yearold children were tested in order for the design and properties of the new digital as- 
sessment tool to be optimized through creating design guidelines. In this project, participants were asked to play eight distinct games in pairs, reporting which game they preferred, to end up to the most favorite game. It was hoped that "DYSL-X" would increase kids' incentives, encouraging them to pay more attention, which would enhance accurate measurement and more reliable evaluation [18].

In Malaysia, Shamsuddin et al. (2017) suggested a feature selection algorithm, which generated classification models dependent on five classifiers (e.g. MultiClassClassifer, J48, ZeroR, Bagging and Bayes Net) for early screening of dyslexia. Specifically, feature selection algorithm functions through detecting sets of characteristics, interdependently associated with the target groups. The dataset was collected from a computer-based screening test, comprised of 50 questions. As algorithms in the form of selection questions were utilized, improvements concerning performance and accuracy of categorization of children with dyslexia were observed. Findings of this paper reported that the attributes were chosen form modules reading, hearing, IQ, spelling and sorting. The greatest accuracy was $100 \%$, implementing Bayes Net as the classifier and CFS as a feature selection algorithm. Nevertheless, there was much scope for additional research looking at further features of this tool by inspecting students' learning profiles [19].

\section{Dyslexia Screening at Primary School}

It is common knowledge that there are both an ideal age and stage of dyslexia to be assessed at primary school, even though the dyslexia detection is no easy task like most learning and teaching [20]. That is to say, the potential signs of dyslexia are perceived in school age, as the child meets the script language, the failure of which acts as a basic symptom of this learning disability. However, the diversion of problems makes it difficult to be detected. Recently, the state-of-the-art equipment of the school and avant-garde ICTs tools optimize the identification of dyslexia.

In particular, Hassan et al. (2017) tried to detect dyslexic children, introducing Malay language mobile game, "Dleksia game", based on different deficit theories. It consists of both data collection module and data analysis module. Firstly, ten distinct mini-games being played by students with the view to collecting useful information about screening, which is responsible for the decision making. In this study, a game usability testing was conducted toward dyslexic children at Ampang in Kuala Lumpur. Most of participants were in favor of this application, feeling that they were playing a game instead of being screen purposely. Unquestionably, the "Dleksia game" seemed to be a distinctive approach for simplifying the dyslexia screening process [10].

Furthermore, Marksa \& Burdenb (2005), also, applied the Cognitive Profiling System (CoPS 1) to investigate the validity of it as a predictor of dyslexia and of measures associated with each student' $\mathrm{s}$ attainment in literacy and mathematics. CoPS 1 is about a psychometric assessment system presented in the form of computer games to toddlers in their early school years with the aim of detecting probable general or specific disabilities. Therefore, a small-scale longitude study was taken place 
among children in two primary schools in the west of England. This investigation produced illuminative findings for the predictive validity of CoPS concerning reading, numeracy and IQ, since it was proved a useful information source especially for students with insufficient reading skills in early school years. Nevertheless, extreme caution ought to have been taken for the successful early diagnosis of dyslexia and learning difficulties, when it was implemented [21].

In Malaysia, Ekhsan et al. (2012) presented a computer-based screening tool, "Smart Lexic", as an alternative method to the manual screening for dyslexics. The development of a new application, which comprised appropriate multimedia elements for dyslexic students, was the aim of this research. Students at early primary school, who tended to be dyslexic according to the observation of their teachers, were tested. In this attempt, both paper-based and multimedia screening tool of the test were utilized. This study highlighted that "Smart Lexic" arised participants' interest and concentration, meeting the users' needs of effective identification of dyslexic children. There is no question, that not only was it user friendly choice but also was a valuable technique in detecting children with dyslexia in early stage [22].

In Malaysia, Isa et al. (2017), also, proposed an innovative computer-based and stand-alone application for detection of dyslexia in school age, named "i-Dyslex". Thus, a heuristic evaluation of it was carried out among experienced expert evaluators, with the view to possible design and interface problems being identified. This tool, using Malay language, features five modules ("Mendengar", "Membaca", "Berfikir", "Mengeja", "Menyusun"), each of which include a 10-question screening dyslexia test. Through offline activities, presentation of tool and positive feedback for further improvement, experts concluded that this application could live up to users' expectations. However, further research might have shed light on dyslexia screening, as the improvements of "i-Dyslex" tool would be taken place and usability testing would be conducted with both dyslexic and non-dyslexic children to get reliable findings [23].

In Singapore, Brooks et al. tried to explore the efficiency of the computerizedbased Lucid Rapid Dyslexia Screening in the detection of students with dyslexia or other literacy difficulties between 2009-2011. The Lucid Rapid depicts a kid's performance in three dyslexia sensitive standards with discrete score, which refer to phonological processing, visual verbal integration, memory/phonic decoding and auditory sequential memory. The exploratory investigation was organized in two phases. Thus, firstly, children were administered the computerized-based tool by trained phycologists and educational therapists at the DAS and subsequently they were undergone full psychological assessments by the aforementioned specialists. Overall, findings ascertained the effectiveness and usefulness of this software for identification of dyslexia, as the majority of children who were assessed to be at risk of dyslexia on the Lucid Rapid were likely to be diagnosed as dyslexic through formal psychological screening too [24].

In Malaysia, Yusoff et al. (2011) devised a Learning Disability Detection System for Autism and Dyslexia, called "Le-ADS", aiding teachers and school principals to categorize possibly autistic and dyslexic children. This software accumulates a great number of assets such as identification of autism and dyslexia in students and laying 
foundation of the appropriate learning curriculums for these types of disorder. In this project, interviews were held, involving experience teachers. Under these conditions useful information was gained, since respondents collaborated with autistic and dyslexic children through educational programs. As a result, it was urgent that the development of this software should have encourage teachers to pay attention on the existence and the importance of early screening of learning problems [25].

In Greece, Zygouris et al. (2016) designed a web screening battery of tests with the aim of exploring elementary students with dyslexia and typically achieving students' orthographic, cognitive and reading skills. Therefore, a great number of right handed kids were distributed this web application, which was based on seven tasks. The results of this investigation reported that children with dyslexia tended to be characterized by lower performance, fewer correct responses and longer latencies in all activities than their average peers. This significant finding seemed to verify the discriminative power of the advanced web tool [26].

In Spain, Bartolomé et al. (2012) come up with a web platform intended for the identification of dyslexia in students at the reading stage. This platform proposes a set of six games, facilitating the diagnosis of characteristics referred to dyslexia. Every game is dedicated to one of the features, containing items, whose degree of difficulty is augmented, as the player makes a progress through the game. Thus, investigating the properties of this system, primary students with probable symptoms of dyslexia were tested and then were compared to their classmates. This paper supported considerable views that web platform could become a successful instrument for evaluation of potential dyslexic children [27].

Additionally, Rauschenberger et al. (2018) introduced a web-based game, named "Mus Vis", which embedded musical and visual cues. The presented web application incorporates two perception games ("sound" and "visual"), that include four stages and eight rounds, triggering sort-term memory and then estimating differences among students with and without dyslexia in various languages. Dyslexic and non-dyslexic students speaking different languages (Spanish, English, German, Catalan) from two Spanish schools and two German schools were tested. Participants were asked to play all the game rounds in their mother tongue at home or at school, when game instructions were translated into the selected language. Indispensable evidence revealed that "Mus Vis" established eight indicators for the discrimination of Spanish children with and without dyslexia and four indicators which were language independent. This web tool seemed to gain ground around the world by easy identification of children with dyslexia, no matter what language they spoke [28].

Afterwards, in Spain, Rauschenberger et al. (2018) suggested an innovative tablet application, called "DGames", to differentiate between dyslexic and non-dyslexic prereaders by assessing toddlers' performance. This application predicts dyslexia through playing with musical and visual cues. Specifically, "DGames" constituted a revision of the game "MusVis", as the new tool was developed from the pilot study and implementation of it. Results showed that both kids and parents were pleased with the content of the game, even though parents of pre-readers mentioned that it was difficult for their children to discriminate the very short and similar sounds of the musical part. There is no doubt that this up-coming game enhanced the dyslexia screening. Howev- 
er, a large-scale study in a great number of languages (e.g. English, Spanish etc.) was needed to validate the effectiveness of the improved model [29].

In Malaysia, Ubaidullah \& Hamid (2012) presented a novel web-based screening instrument, known as "e-ISD", for identifying dyslexic students in a more accurate and quicker way, compared to the manual-based screening process. Specifically, at the preliminary stage of this project, a great number of semi-structured interviews were organized, in which teachers and a school principal from an elementary school participate, prior to designing the desirable prototype. Results came to light a need for the development of a new system, that could improve the detection process. Interviewees were excited about the proposed application, which was believed to accumulate vital characteristics and functionalities for the evaluation of dyslexia. Hence, further analysis was needed, taking into consideration the development of a robust framework to perform all the screening tasks [30].

In Australia, Rauschenberger et al. (2017) presented an innovative application, named "DysMusic", which was intent on evaluating discrepancies through the perception of auditory elements, caused by difficulties in short-term memory, before acquiring basic reading skills. This language independent system is structured by four activities without interruptions, each of whom encompasses two subtasks with a significant number of cards and sound files. Inevitably, a test usability was conducted among German native children, who were accompanied by their parents. All participants, having played the memory game with musical characteristics carefully over the website, were totally delighted with game elements. "DysMusic" was recognized as an easy and entertaining tool, as the subjects made progress in distinguishing the sounds and choosing the right card pairs in accordance with the musical elements after the first activities [31].

In Malaysia, Che-Pee et al. (2016) designed a Cross-Platform Dyslexia Screening Test, known as "Dycsreen", for the assessment of dyslexia in primary students through hybrid applications. "DycScreen" includes five categories associated with dyslexia symptoms, which are composed of questionnaires, referred to vision, spelling, mathematics, direction time and cognitive skills. In this project, a tutor and a great number of dyslexic and non-dyslexic students were tested, answering all questions in each category. Observations demonstrated that the system identified potentially dyslexics in an adequate way. All subjects were willing and more interested in taking part in screening test via digital technologies, although they were struggled in certain assessments (e.g. cognitive assessments). It was hoped that more cases of children with dyslexia would be evaluated thanks to success of this computerized method [32].

In Boston, Hassanain (2017) envisioned a tablet-based and big data-based multimedia environment, so that the process of identifying dyslexia could be accelerated and be optimized through collecting a wide variety of data for in-depth dyslexia pattern analysis. This advanced tool features four test modules (Clock Drawing Test, Writing Test, Reading Test, Drawing Family Members), using text video, gaze and audio movement to evaluate attributes of dyslexia among children. The test modules were tested with a Samsung Tablet, when other properties of this system were approved or were taken under consideration by using special algorithms. Overall, as far 
as the usability of the multimedia environment was concerned more research on the performance of it on a national level was needed [33].

In Malaysia, Hanif-Jofri (2018) developed a dyslexia screening tool dependent on a mobile application, called "Kiddo Disleksia". The application deploys the power of video, text, graphic and audio in multimedia. Dyslexic primary students were detected with auditory and visual techniques, through recognizing and discriminate the alphabet letter and phonic. Thus, a usability study was carried out among pupils with dyslexia. As highlighted, there were significant differences between dyslexics with mild and severe symptoms concerning their speed in completing tasks, as well as reading recognition and comprehension. Spelling and listening, in contrast, were not vital discrepancy indicator for them. Inevitably, most of participants commented that "Kiddo Disleksia" offered an easy and enjoyable way to learn without understanding the screening process [34].

In the U.K., Kohli (2010) presented "Artificial Neural Networks" to scientific community as an alternative solution for the identification of students with dyslexia. "Artificial Neural Networks" constitute devices, implemented by utilizing electronic components or simulated in software on a computer, that monitor the way in which the brain performs a function or task of interest. Hopeful results of this preliminary study reported that "Artificial Neural Network" were a sufficient model to detect dyslexic children. Hence, efforts and supplementary related researches on students' difficulties were required, in order for the new tool to be characterized by high accuracy [35].

In South Australia, Peters et al. (2007) implemented QEEG and neuropsychological tests to look into the veiled neural processes in dyslexics compared to nondyslexic students. Specifically, a group of children and a matched control group from the Brain Resource International Database were targeted on indicators of brain function and cognition (EEG and coherence). EGG coherence constitutes a measure, which displays functional connectivity between brain areas and deviation of this activity. Research data reported remarkable discrepancies in two groups on verbal interference tests and the memory recognition test. Also, increased slow activity in the frontal and right temporal regions of the dyslexics' brain and differences in operations of other related regions like possible compensatory mechanisms were observed, as opposed to control group. Indisputably, this paper supported that neurobiological causes underlie dyslexia, contributing to the diagnosis of this learning disability [36].

In Saudi Arabia, Al-Barhamtoshy et al. (2017) proposed an emergent computing system, framed by computational classifiers (K-means, ANN, Fuzzy) to diagnose dyslexia. This digital tool, aimed at individual' s problems with spelling, reading, speaking and writing, categorized the dataset into three classes, that were "dyslexic" or "normal (none dyslexic)", or "other disorders (e.g. hyperactivity). Numerous children, aged from 7 to 13 years old, were examined through implementing the three selected classifiers. Classifiers measurements of the data items of the dataset revealed that the factors, which had the primary effect, were auditory and visual processing, as well as word attack and reading strategy. Indeed, computing analysis strategies were expected to be both efficient and accurate system at a remarkable extent in process of dyslexia screening [37]. 


\section{Dyslexia Screening in Adulthood}

According to Reynolds (2014) a noticeable proportion of students are not detected as dyslexics in childhood, which is feasible only when they become a part of postsecondary institutions [38]. This situation has detriment effects on individuals' adaptation in everyday life, as well as the community. ICT tools have provided a real breakthrough in the recent years, helping dyslexic people to realize and cope with their difficulty irrespective of their age.

Particularly, Singleton et al. (2009) implemented three computer-based tests, "Word Recognition test", "Word Construction test" and "Working Memory test", which focus mainly on working memory, phonological processing and lexical access. Examining the validity of the computer-based approach for the detection of dyslexia in adulthood, these tools were tested to dyslexic and non-dyslexic adults from three different types of educational institution in the UK. The results revealed that the former and the latter were substantially differed in reading and spelling abilities. Furthermore, adaptive versions of the computer-based tests were developed, showing that combined scaled scores of them discriminated the dyslexic from the non-dyslexic group to a significant extent. This method was expected to offer a practical solution for the identification of dyslexia in adults [11].

In Bahasa Melayu, Hazawawi \& Hisham (2014) designed an online screening test, known as "MaDIST". This web tool is composed of a questionnaire about participant' s possible learning difficulties history and a reading assessment test. Malaysian young adults were targeted, in order for useful information to be collected about the detection of potentially dyslexic users. In seeking to improve the screening process, researchers realized that some participants had learning difficulties throughout their school years (e.g. reading problems), which indicated that reading assessment was also a factor of primary importance in diagnosing dyslexia among young adults [39].

Moreover, Nichols et al. (2009) compared a tutor-delivered battery of 15 subtests for dyslexia, dyspraxia and Meares-Irlen (M-I) syndrome with a computerized test, known as "Lucid Adult Dyslexia Screening test (LADS)" at University of Worcester. LADS, based on an algorithm, gives the opportunity to weight student's performance on its subtests of word recognition, memory and word construction against his performance on its nonverbal reasoning test. As outlined, a great number of volunteer students were diagnosed with at least one of the difficulties under investigation, the majority of whom were dyslexic. The outcomes of this study suggested that combination of LADS and four of the screening original tutor delivered subtests should be the most valuable option, gaining ground for dyslexia [40].

Costa et al. (2013) presented a computational tool, "DysDTool", which was depended on neural networks for the evaluation of learning difficulties such as dyslexia. It is about a multi-layer system with several models, which is characterized by extended spectrum of difficulty in its content (letters, numbers, games etc.). The target group consisted of dyslexic and non-dyslexic students aged from 9 to 18 years old in Rio de Janeiro (Brazil) and Salamanca (Spain). For this purpose, the student, accompanied by their parents and legal guardians, participated in private interviews that were occurred at home, school and social areas. Empirical comparisons and observa- 
tions of the accuracy of the tool revealed the outstanding advantage of it to categorize people with or without dyslexia, affirming the durability of it in diagnosis of dyslexia [41].

In Mexico, Rello et al. (2016) recommended an easy to access linguistic web-based game, called "Dytective", so as they could identify differences between children and adults with or without dyslexia. This application is composed of 17 timed stages, consisted of exercises related to linguistic and attentional skills with increased difficulty rate, which screen dyslexia through discrepancies in dyslexics' and nondyslexics' scores. Experimental study was conducted among dyslexic and nondyslexic kids and adults, who are asked to play all levels of the game by parental consent. The results showed that this tool constituted a capable predictable indicator of screening the risk of dyslexia with accuracy regardless of the distribution of individuals [42].

In Spain, Mejía et al. (2012) presented a new computerized assessment battery for detecting adults with dyslexia, ("BEDA"), which aimed to evaluate mainly the deficient cognitive processes, using auditory, visual and speech communication channels. The system comprises of 8 modules, which referred to cognitive process, analysis of results and administration purposes. Both teachers and students participated in the case study, completing the "BEDA" items at home or in computer labs in their university, in order for usability and discriminate validity of the tool to be measured. On the one hand, students were satisfied with "BEDA" tasks, although they had difficulty in understanding the audio guidelines of some activities. On the other hand, teachers were not well informed of dyslexic students. Unquestionably, utilizing "BEDA" in a learning-teaching environment may have been useful in terms of helping individuals to identify their deficient cognitive processes and confirming the dyslexia diagnosis [43].

In addition, Takács et al. (2010) constructed a computerized test battery, "HAD", intending to evaluate symptoms of dyslexia in Hungarian adult population. "HAD" test consists of twelve assessment modules, which are mainly oriented towards reading, writing and supplementary executive functions related to these significant aspects of written discourse. In this study, control and dyslexic groups were targeted, being urged to complete the computer assisted test battery. Current analysis of the study showed that tow selected groups were differentiate in most tests. Hence, there are not any discrepancies in general cognitive abilities (e.g. visual memory, visual special memory, executive functions etc.), inspecting controls' and dyslexics' skills. It was supported that "HAD" may represent a valid assessment tool for high, moderate and low risk of dyslexia in adulthood [44].

In Italy, Rello \& Ballesteros (2015) implemented a system with eye tracking measures, known as "Support Vector Machine binary classifier" to classify effectively individuals with dyslexia or without dyslexia. In this eye tracking experiment, a great number of dyslexics and non-dyslexics with normal or corrected-to-normal vision, aged from 11 to 50 years old participated. Thus, subjects were asked to read recorded readings of each opted text, as the eye tracker was incorporated on them individually. Obviously, this unique assessment technique detected automatically people with dys- 
lexia readings with great accuracy, using the most informative features (e.g. mean of fixation, reading time, age of participants etc.) [45].

In Portugal, Mejía-Corredor et al. (2011) presented a web-based system, known as "DetectLD", to identify university students with learning disabilities such as dyslexia. "DetectLD", based on a self-questionnaire for the screening of learning disabilities in reading or writing, supports different self-questionnaires. Also, it is composed of four modules which are administrator module, expert module, teacher module, student module). In this study, undergraduate and graduate students from University of Girona were tested, so as for performance and usability of it to be verified. Data analysis revealed that the new tool was appealing, related to content, as well as easy and quick to fill in and understand, that sealed the success and improvement of it through students' and teachers' useful comments and experiences [46].

\section{Conclusion}

In conclusion, the sooner a dyslexic student is detected, the better results are gained so as for potential pertinent disorders and other possible problems to be dealt with effectively. Nowadays, technology has developed magnificent tools and resources, addressed to all ages, to optimize the conditions of the screening dyslexia process. In this endeavor, the selection of the appropriate screening tool seems to play a critical role, since it should meet the required criteria in relation to the needs of targeted group. Therefore, the researcher or teacher ought to take into account numerous factors of the provided system (e.g. validity, reliability etc.) with the view to desired information being retrieved, as well as the results being undeniable. Hence, several research areas are needed to be investigated concerning the usability, implementation and potential adaptation of some ICT tools. The results of this review are really hopeful, as ICT tools, combined with other detection methods expected to be as a key solution for ensuring and reinforcing the identification of dyslexia.

\section{$6 \quad$ References}

[1] G. R. Lyon, S. E. Shaywitz, and B. A Shaywitz, "Defining Dyslexia, Comorbidity, Teacher's Knowledge of Language and Reading: A Definition of Dyslexia," Annals of Dyslexia, vol. 53, pp. 1-14, 2003. https://doi.org/10.1007/s11881-003-0001-9

[2] P. Juneja, "Dyslexia: Assessment and problem faced," Indian Journal of Health and Wellbeing, vol. 9, no. 2, Feb., pp. 308-311, 2018.

[3] R. L. Peterson and B. F. Pennington, "Developmental Dyslexia," The Annual Review of Clinical Psychology, vol. 11, no. 9, Jan., pp. 9.1-9.25, 2015.

[4] M. J. Snowling, "Early identification and interventions for dyslexia: A contemporary view," Journal of Research in Special Educational Needs, vol. 13, no. 1, Jan., pp. 7-14, 2013. https://doi.org/10.1111/j.1471-3802.2012.01262.x

[5] J. Worthy, S. DeJulio, N. Svrcek, D. A.Villarreal, C. Derbyshire, K. LeeKeenan, M. T. Wiebe, C. Lammert, J. C. Rubin, and C. Salmerón, “Teachers' understandings, perspectives, and experiences of dyslexia," Literacy Research: Theory, Method, and Practice, vol. 65, no. 1, Nov., pp. 436-453, 2016. https://doi.org/10.1177/2381336916661529 
[6] H. Perera, M. F. Shiratuddin, and K. W. Wong, Review of the Role of Modern Computational Technologies in the Detection of Dyslexia. In Kim, K. J. \& Joukov, N. (eds). Information Science and Applications (ICISA). Spinger Science+ Business Media: Signapore, 2016.https://books.google.gr/books? id=rZKVCwAAQBAJ\&pg=PA1464\&lpg=PA1464\&d $\mathrm{q}=\% \mathrm{E} 2 \% 80 \% 9 \mathrm{CReview}+\mathrm{of}+$ the + Role + of + Modern + Computational + Technologies + in + the + Detection + of + Dyslexia\%E2\%80\%9D. + In + Kim,+ K. + J. $+\% 26+$ Joukov, + N..$+($ eds $) .+$ Inform ation+Science+and+Applications\&source. https://doi.org/10.1007/978-981-10-0557-2 141

[7] M. Rooms, Information and Communication Technology and Dyslexia. In Towned, J. \& Turner, M. (eds). Dyslexia in Practice: A Guide for Teachers. (pp. 263-272). Springer Science+Business Media: New Yorks, 2000. https://www.springer.com/gp/book/978030646 2511. https://doi.org/10.1007/978-1-4615-4169-1 11

[8] C. Singleton, "Computer-based assessment in education," Educational and Child Psychology (Special Issue on Information Technology and Children's Learning), vol. 18, no. 3, pp. 58-74, 2001. https://pdfs.semanticscholar.org/5205/08ebad396609eeda2d071a73f7a413b 77e5f.pdf. [Accessed: December 23, 2018]

[9] K. Kalyvioti, and T. A. Mikropoulos, "Virtual Environments and Dyslexia: A literature review," Procedia Computer Science, vol. 27, pp. 138 - 147, 2014. https://doi.org/10.1016/j. procs.2014.02.017

[10] Z. Hassan, S. Mohtaram, N. Che Pee, and A. S. Shibhatulah, "Dyslexia Game: A Mobile Dyslexia Screening Test Game to Screen Dyslexia Using Malay Language Instruction," Asian Journal of Information Technology, vol. 16, no. 1, pp. 1-6, 2017.

[11] C. Singleton, J. Horne, and F. Simmons, "Computerized screening for dyslexia in adults," Journal of Research in Reading, vol. 32, no 1, Jan., pp 137-152, 2009. https://doi.org/10. 1111/j.1467-9817.2008.01386.x

[12] H. W. Catts and Y. Petcher, "Early Identification of Dyslexia: Current Advancements and Future Directions," Perspectives on Language and Literacy, vol. 44, no. 3, pp. 33-36, 2018. [Online serial] Available: https://shanahanonliteracy.com/upload/publications/ 163/pdf/ed3a5412095be4320e550941575ced4b3682086e.1.pdf\#page=33 (Accessed: December 23, 2018)

[13] M. R. Sood, A. Toornstra, M. I. Sereno, M. Boland, D. Filaretti, and A. Sood, "A Digital App to Aid Detection, Monitoring, and Management of Dyslexia in Young Children (DIMMAND): Protocol for a Digital Health and Education Solution", JMIR Research Protocols, vol. 7, no. 5, May, pp. 135-142, 2018. [Online serial] Available: https://www.ncbi.nlm.nih.gov/pmc/articles/PMC5981053/ (Accessed: December 23, 2018) https://doi.org/10.2196/preprints.9583.a

[14] N. S. F. N., Mat, S. N. W. Shamsuddin, R. b. Husain, M. Makhtar, W. M. W. Isa, and F. S. Mohamad, "A Conceptual Framework for Designing a Computer-based Dyslexia Screening Test," In Proc. of the Third International Conference on Informatics \& Applications, 2014, pp. 46-50.

[15] O. Gaggi, G. Galiazzo, C. P. Padua, A. Facoetti, and S. Franceschini, “A Serious Game for Predicting the Risk of Developmental Dyslexia in Pre-readers Children,”. In Proc. of 21st International Conference on Computer Communications and Networks (ICCCN), 2012, pp. 1-5. https://doi.org/10.1109/iccen.2012.6289249

[16] V.T. Bai, P.M.B. Dr Devamalar, M.A. Mr Marx, and G. Dr. Kavya, "An interactive Fine motor Movements Screening e-Tool for Dyslexia based on Kinesthetic Perception," In Proc. of 2nd International Conference on Advances in Electrical, Electronics, Information, Communication and Bio-Informatics (AEEICB), 2016, pp. 1-4. https://doi.org/10.1109/ aeeicb.2016.7538294 
[17] A. Facoetti, S. Franceschini, O. Gaggi, G. Galiazzo, S. C. Gori, E. Palazzi, M. Ruffino Franceschini, O. Gaggi, G. Galiazzo, S. Gori, C. E. Palazzi, and M. Ruffino, "Multiplatform Games for Dyslexia Identification in Preschoolers," In Proc. of 11th Consumer Communications and Networking Conference (CCNC), 2014, pp. 1152-1153. https://doi.org/10.1109/ccnc.2014.6994419

[18] L. V. Den Audenaeren, V. Celis, V. V.,Abeele, L. Geurts, J. Husson, P. Ghesquière, J. Wouters, L. Loyez, and A. Goeleven, DYSL-X: Design of a tablet game for early risk detection of dyslexia in preschoolers. In B. Schouten et al. (eds.). Games for Health, Springer: Fachmedien, Wiesbaden 2013. [E-Book] Available: https://core.ac.uk/download/ pdf/34589748.pdf https://doi.org/10.1007/978-3-658-02897-8_20

[19] S. N. W. Shamsuddin, N. S. F. N. Mat, and M. Makhtar, "Relevant Test et Using Feature Selection Algorithm for Early Detection of Dyslexia," Journal of Fundamental and Applied Sciences, vol. 9, no. 6S, pp. 886-899, 2017. https://doi.org/10.4314/jfas.v9i6s.66

[20] M. Houston, Identification of Dyslexia at Primary School, In Supporting Pupils with Dyslexia at Primary School, no. 4, Dyslexia Scotland: Great Britain, 2011. [E-Book] Available: http://training.cpdbytes.com/ResourceFiles/All/P4IdentificationOfDyslexiaAtPrimary. pdf

[21] A. Marksa, and B. Burden, "How Useful are Computerised Screening Systems for Predicting Subsequent Learning Difficulties in Young Children? An Exploration of the Strengths and Weaknesses of the Cognitive Prof iling System (CoPS 1)," Educational Psychology in Practice, vol. 21, no. 4, Dec., pp. 327-342, 2005. https://doi.org/10.1080/026673605003 $\underline{44971}$

[22] H. M. Ekhsan, S. Z. Ahmad, S. A. Halim, J. N. Hamid, N. H. Mansor, "The implementation of interactive multimedia in early screening of dyslexia," In Proc. of 2012 International Conference on Innovation, Management and Technology Research (ICIMTR2012), 2012, pp. 566-569. https://doi.org/10.1109/icimtr.2012.6236459

[23] W. M. W. Isa, S. N. W., Shamsuddin, N. S. F. N. Mat, M., Makhtar, F. S. Mohamad, and R. Husain, "Heuristic Evaluation Of i-Dyslex Tool for Dyslexia Screening," Journal of Telecommunication, Electronic and Computer Engineering, vol. 9, no. 2-4, pp. 15-19, 2017.

[24] G. Ng. V. Brooks, B. H. Lim, W. P. Tan, and N. Lukito, "The Computerised-based Lucid Rapid Dyslexia Screening for the Identification of Children at Risk of Dyslexia: A Signapore study," Educational and Child Psychology, vol. 28, no. 2, pp. 119-144, 2015.

[25] N. M. Yusoff, N. S. Rusli, R. Ishak, and E. A. Jabbar, "LeADS: Early Learning Disability Detection System for Autism and Dyslexia," In Proc. of 14th International Conference on Human-Computer Interaction (HCI) - Posters' Extended Abstracts, 2011, pp. 433-437. https://doi.org/10.1007/978-3-642-22095-1_87

[26] N. C. Zygouris, F. Vlachos, A. N. Dadaliaris, P. Oikonomou, G. I. Stamoulis, D. Vavougios, E. Nerantzaki, and A. Striftou, "The Implemenation of a Web Application for Screening Children with Dyslexia," In Proc. of $19^{\text {th }}$ International Conference on Interactive Collaborative Learning, ICL, 2016, pp. 415-423. https://doi.org/10.1109/icl.2015.7318103

[27] N. A. Bartolomé, A. M. Zorrilla, and B. G. Zapirain, "Dyslexia diagnosis in Reading Stage though the use of games at school," In Proc. of the 17th International Conference on Computer Games (CGAMES), 2012, pp. 12-17. https://doi.org/10.1109/cgames.2012.6314 $\underline{544}$

[28] M. Rauschenberger, L. Rello, R. B. Yates, and J. P. Bigham, "Towards Language Independent Detection of Dyslexia with a Web-based Game," In Proc. of the Internet of Accessible Things, 2018, pp. $\chi \chi$. https://doi.org/10.1145/3192714.3192816 
[29] M. Rauschenberger, L. Rello, R. Baeza-Yates, E. Gomez, and J. P. Bigham, "Towards the Prediction of Dyslexia by a Web-based Game with Musical Elements," In Proc. of the 14th Web for All Conference on the Future of Accessible Work, 2017, pp. 1-4. https://doi.org/10.1145/3058555.3058565

[30] H. H. Bt. Ubaidullah and J. Hamid, "A web-based screening system for dyslexic pupils: Do teachers need it?" I-manager's Journal on Educational Psychology, vol. 5, no. 4, Feb.April, pp. 15-23, 2012. https://doi.org/10.26634/jpsy.5.4.1685

[31] M. Rauschenberger, L. Rello, and R. Baeza-Yates, "A Tablet Game to Target Dyslexia Screening in Pre-Readers," In Proc. of the 20th International Conference on HumanComputer Interaction with Mobile Devices and Services Adjunct (MobileHCI'18), 2018, pp. 306-312. https://doi.org/10.1145/3236112.3236156

[32] N. Che Pee, P. H. Leong, M. A. Othman, H. A. Sulaiman, M. F. I. Othman, and Y. A. Rahim, "DycScreen-Cross-Platform Dyslexia Screening Test for Malaysian Children Through Hybrid Applications," In Proc. of ICOCOE 2015, 2016, pp. 1083-1091. https://doi.org/10.1007/978-3-319-24584-3 92

[33] E. Hassanain, A Multimedia Big Data Retrieval Framework to Detect Dyslexia Among Children. In Proc. of 2017 IEEE International Conference on Big Data (BIGDATA), 2017, pp. 3857-3860. https://doi.org/10.1109/bigdata.2017.8258389

[34] M. H. Jofri, "TOPIC: Kiddo Disleksia Mobile Application: A Dyslexia Screen Tool in Malay Language," In Proc. of the International University Carnival on e-Learning (IUCEL) 2018, 2018, pp. 609-612.

[35] M. Kohli, "Identifying Dyslexic Students by Using Artificial Neural Networks," In Proc. of the World Congress on Engineering 2010 (WCE 2010), 2010, pp. $\chi \chi$.

[36] A. M. Peters, S., R Breteler, and L. Verhoeven, "Different Brain Activation Patterns in Dyslexic Children: Evidence from EGG power and Coherence Patterns for the DoubleDeficit Theory of Dyslexia," Journal of Integrative Neuroscience, vol. 6, no. 1, pp. 175190. 2007. https://doi.org/10.1142/s0219635207001404

[37] H. M. Al-Barhamtoshy and D. E. M. Motaweh, "Diagnosis of Dyslexia using Computing Analysis," Journal of Engineering Technology, vol. 6, no. 2, Jul., pp. 462- 482, 2017.

[38] A. Reynolds, "Dyslexia in Adulthood: Screening Assessment and Manifestations," in Thesis submitted in fulfilment of the requirements for the degree of Doctor of Philosophy. UK: Bangor University, [online document], 2014. Available: http://e.bangor.ac.uk/5046/1/ Andrea\%20Reynolds\%20PhD.\%20Thesis.pdf [Accessed: Dec: December 23, 2018].

[39] N. A. M. Hazawawi and S. Hisham, "Online dyslexia screening test for Malaysian young adults in Bahasa Melayu," In Proc. of 5th International Conference on Information and Communication Technology for The Muslim World (ICT4M), 2014, pp. 1-5. https://doi.org/10.1109/ict4m.2014.7020676

[40] S. A. Nichols, J. S. McLeod, R. L. Holder, and H. S. T. McLeod, Screening for Dyslexia, Dyspraxia and Meares-Irlen Syndrome in Higher Education. DYSLEXIA, vol. 15, no. 1, Feb., pp. 42-60, 2009. https://doi.org/10.1002/dys.382

[41] M. Costa, J. Zavaleta, S. M. Serra da Cruz, M. Manhães, R. Cerceau, L. A. Carvalho, and C. R. Mousinho, "A Computational Approach for Screening Dyslexia," In Proc. of the 26th IEEE International Symposium on Computer-Based Medical Systems, 2013, pp. 565569. https://doi.org/10.1109/cbms.2013.6627879

[42] L. Rello, M. Ballesteros, A. Ali, M. Serra, D. Alarcón-Sánchez, and J. P. Bigham, "Dytective: Diagnosing Risk of Dyslexia with a Game," In Proc. of the 10th EAI International Conference on Pervasive Computing Technologies for Healthcare, 2016, pp. 89-96. https://doi.org/10.4108/eai.16-5-2016.2263338 
[43] C. Mejía, A. Diaz, J. E. Jiménez, and R. Fabregat, "BEDA: a computerized assessment battery for dyslexia in adults," Procedia - Social and Behavioral Sciences, vol. 46, pp. 1795 1800, 2012. https://doi.org/10.1016/j.sbspro.2012.05.381

[44] J. Takács, A. Lukács, B. Kas, I. Kóbor, V. Gál, and Z. Vidnyánszky, "HAD test: a new Hungarian adult dyslexia screening test," Front. Neurosci. Conference Abstract: IBRO International Workshop, vol. $\chi$, no. $\chi$, p. $\chi$. May 2010. [Abstract]. Available: https://www.frontiersin.org/10.3389/conf.fnins.2010.10.00187/event abstract [Accessed December 23, 2018]. https://doi.org/10.3389/conf.fnins.2010.10.00187

[45] L. Rello and M. Ballesteros, "Detecting Readers with Dyslexia Using Machine Learning with Eye Tracking Measures," In Proc. of the 12th Web for All Conference, 2015, pp. $\chi \chi$.

[46] C. M. Corredor, J. C. Marquez, R. F. Gesa, "DetectLD: Detecting University Students with Learning Disabilities in Reading and Writing in the Spanish Language,". In Proc. EDMEDIA 2011-World Conference on Educational Multimedia, Hypermedia \& Telecommunications, 2011, pp. 1122-1131. https://doi.org/10.1145/2745555.2746644

\section{Authors}

Athanasios Drigas is with N.C.S.R. 'Demokritos', Institute of Informatics and Telecommunications, Net Media Lab, and Mind-Brain R \& D, Agia Paraskevi, 153 10, Athens, Greece (e-mail: dr@,iit.demokritos.gr).

Stratigoula Politi-Georgousi is currently working as a special education teacher in Athens, Greece. She holds a Master' s degree in Special Education and ICTs (e-mail: stratigoulaplt@gmail.com).

Article submitted 2019-06-10. Resubmitted 2019-07-16. Final acceptance 2019-07-24. Final version published as submitted by the authors. 